\title{
Ten-Year Cancer Incidence in Rescue/Recovery Workers and Civilians Exposed to the September 11, 2001 Terrorist Attacks on the World Trade Center
}

\author{
Jiehui Li, MBвs, Ms, ${ }^{1 *}$ Robert M. Brackbill, PhD, MPH, ${ }^{1}$ Tim S. Liao, MPH, ${ }^{1}$ Baozhen Qiao, PhD, $^{2}$ \\ James E. Cone, MD, MPH, ${ }^{1}$ Mark R. Farfel, ScD, ${ }^{1}$ James L. Hadler, MD, MPH, ${ }^{1}$ Amy R. Kahn, ${ }^{\text {Ms, }}{ }^{2}$ \\ Kevin J. Konty, MS, MA, ${ }^{1}$ Leslie T. Stayner, $\mathrm{PhD},{ }^{3}$ and Steven D. Stellman, PhD, MPH ${ }^{1,4}$
}

\begin{abstract}
Background Cancer incidence in exposed rescue/recovery workers (RRWs) and civilians (non-RRWs) was previously reported through 2008.

Methods We studied occurrence of first primary cancer among World Trade Center Health Registry enrollees through 2011 using adjusted standardized incidence ratios (SIRs), and the WTC-exposure-cancer association, using Cox proportional hazards models.

Results All-cancer SIR was 1.11 (95\% confidence interval (CI) 1.03-1.20) in RRWs, and 1.08 (95\% CI 1.02-1.15) in non-RRWs. Prostate cancer and skin melanoma were significantly elevated in both populations. Thyroid cancer was significantly elevated only in RRWs while breast cancer and non-Hodgkin's lymphoma were significantly elevated only in non-RRWs. There was a significant exposure dose-response for bladder cancer among RRWs, and for skin melanoma among non-RRWs.

Conclusions We observed excesses of total and specific cancers in both populations, although the strength of the evidence for causal relationships to WTC exposures is somewhat limited. Continued monitoring of this population is indicated. Am. J. Ind. Med. 59:709-721, 2016. (c) 2016 Wiley Periodicals, Inc.
\end{abstract}

KEY WORDS: cancer incidence; environmental exposure; World Trade Center; September 11 attacks

\footnotetext{
${ }^{1}$ World Trade Center Health Registry, New York City Department of Health and Mental Hygiene, Long Island City, New York

${ }^{2}$ Bureau of Cancer Epidemiology, New York State Department of Health, Albany, New York

${ }^{3}$ Division of Epidemiology and Biostatistics, University of Illinois, Chicago, Illinois

${ }^{4}$ Department of Epidemiology, Mailman School of Public Health, Columbia University, New York, New York

${ }^{*}$ Correspondence to: Jiehui Li, MBBS, MS, New York City Department of Health and Mental Hygiene, 42-09 28th Street, Queens, NY 11101. E-mail: jli3@ health.nyc.gov

Accepted 11 July 2016

DOI 10.1002/ajim.22638. Published online in Wiley Online Library (wileyonlinelibrary.com).
}

\section{INTRODUCTION}

The attacks of September 11, 2001, on the World Trade Center (WTC) in New York City (NYC) resulted in collapse of the WTC office towers, and an immense cloud of dust and debris that exposed hundreds of thousands of individuals to numerous hazardous substances, including known carcinogens such as asbestos, silica, polycyclic aromatic hydrocarbons (PAHs), benzene, heavy metals (e.g., cadmium, lead), as well as polychlorinated biphenyls (PCBs) [NIOSH, 2011, 2012; Lauby-Secretan et al., 2013].

Because of the fine particulate composition of the dust, combustion products from fires or burning, and the physical forces resulting in its dispersion in the area surrounding 
"Ground Zero" [Lioy et al., 2002, 2006; Landrigan et al., 2004] many individuals experienced intense exposures within the dust cloud and immediately afterwards, less intense exposures over a protracted period of time, or a combination. Rescue and recovery workers were potentially highly exposed between September 11, 2001, and termination of the cleanup in July, 2002, particularly during the immediate response. Half of the workers wore no respiratory protection on the 1st day of response and another one-third wore unrated disposable masks only [Antao et al., 2011]. Thousands of others continued to either inhabit residences and/or work near Ground Zero, or else evacuated and then returned to buildings that underwent prolonged, and sometimes incomplete decontamination.

Previously we reported excess incidence of multiple myeloma, prostate, and thyroid cancer among rescue/recovery workers during 2007 through 2008 [Li et al., 2012]. Increased cancer incidence has also been reported in two cohorts of exposed responders followed through 2008 [Zeig-Owens et al., 2011; Solan et al., 2013]. While total cancer rates were not significantly greater than expected in any of these studies [Zeig-Owens et al., 2011; Li et al., 2012; Solan et al., 2013], each reported a significant increase for at least one sitespecific cancer [Boffetta et al., 2016]. All three cohorts reported a significant two- to threefold increase in thyroid cancer. Comparable increases in prostate cancer incidence were reported by the Fire Department of New York (FDNY) and the World Trade Center Health Registry (WTCHR) [ZeigOwens et al., 2011; Li et al., 2012]. A deficit of lung cancer cases was reported in all three cohorts. However, no excess cancers were reported among community members not involved in rescue/recovery [Li et al., 2012]. A recent study of NYC Police Department personnel compared incidence rates before and after $9 / 11$, and reported a $44 \%$ increase in the overall median age-adjusted incidence rate for total cancers as well as some cancer sites; however, the association between WTC exposure and subsequent cancer development was not examined [Kleinman et al., 2015].

In this report, we continued evaluating possible excess cancer among WTCHR enrollees with three additional years of follow-up, allowing for up to 10 years of latency since the initial exposures, which is important for assessing a possible link to cancer. We recalibrated the definition of WTC disaster physical exposures emphasizing exposure to potential contaminants containing carcinogens as a result of the 9/11 attacks and aftermath [Landrigan et al., 2004].

\section{MATERIALS AND METHODS}

\section{Study Population}

Details of the WTCHR have been published elsewhere [Farfel et al., 2008; Brackbill et al., 2009]. Briefly, in 2003-2004 a total of 71,431 persons enrolled by completing a telephone $(95 \%)$ or in-person (5\%) interview. Participants were either identified through lists provided by employers, government agencies, and other entities (30\%) ("list-identified"), or they responded to an outreach campaign (70\%) ("self-identified") and were enrolled if eligible. Coverage of the eligible exposed population was estimated at $34 \%$ of rescue/recovery workers and $26 \%$ of residents [Murphy et al., 2007]. This analysis included enrollees who lived in any of the 11 states selected for cancer registry linkage (New York, New Jersey, California, Connecticut, Florida, North Carolina, Massachusetts, Ohio, Pennsylvania, Texas, and Washington) from September 11, 2001 through December 31, 2011; 91\% of enrollees lived in these states during the follow-up period. Enrollees had to be at risk for a first primary invasive cancer, defined as never having had a primary invasive cancer documented in any of the 11 state cancer registries.

We assumed, as in our previous report [Li et al., 2012], that any exposure-related cancers would be more likely to emerge at least 5 years after 9/11. The present analysis focused on cancers occurring from 2007 through 2011, restricted to enrollees alive on January 1, 2007 and at risk for a first primary invasive cancer or in situ bladder cancer. Enrollees with unknown ethnicity or unclassifiable race were excluded because reference cancer rates are not available.

The study was approved by the Institutional Review Board (IRB) of the NYC Department of Health and Mental Hygiene. Each cancer registry record linkage was also approved by the respective IRB of 10 state departments of health and the Rutgers University in New Jersey. A Federal Certificate of Confidentiality was obtained, and oral informed consent was obtained from participants at enrollment.

\section{Outcome Ascertainment}

An incident cancer case in our study was a first primary invasive cancer or in situ bladder cancer that had been reported to any of the 11 cancer registries and diagnosed any time post-enrollment through 12/31/2011, the last date for which complete cancer incidence records were available. Cancer site was defined using the Surveillance, Epidemiology, and End Results (SEER) site recode International Classification of Diseases for Oncology, Third Edition (ICD-O-3) groupings, in which categories are based on primary site and histology of cancer [NCI, 2003].

\section{WTC Exposure and Covariates}

Demographic and exposure data were obtained at Registry enrollment. Enrollees were divided into rescue/recovery 
workers (RRW), and civilians or enrollees not involved in rescue/recovery (non-RRW). RRW were first responders, volunteers, and others who worked at the WTC site, debris loading sites or transport barges, or at the Staten Island landfill between September 11, 2001 and June 30, 2002. NonRRW were residents, children, and staff in schools (pre-kindergarten-12th grade) south of Canal Street, area workers and passersby south of Chambers Street on 9/11 in lower Manhattan. Individuals who were in both groups were categorized as RRW.

We defined WTC exposures separately for RRW and non-RRW by focusing on environmental exposures at the WTC site, and lower Manhattan (see Table SI). Among RRW, a WTC exposure matrix was created based on date of arrival, duration of work at the site, dates or time period working on the pile, and being near the WTC site or exposure to dust/debris resulting from the collapse of buildings on 9/11. A modified Delphi Technique [Hasson et al., 2000] was used to arrive at component weighted scores for each exposure item. A scale of 1 (lowest) to 10 (highest) was assigned to each exposure item by a panel of industrial hygienists and occupational disease epidemiologists, and the final set of scores was used to compute a mean score for each item. The sum of WTC exposure scores for each individual rescue/recovery worker was analyzed both as a continuous variable (actual sum scores, and natural log transformed to reduce skew) for trend and as an ordinal scale variable for analysis of cancer risk at each exposure quartile. The latter, based on quartiles of the summed points, categorized as: low (Q1 $<25 \%)$, intermediate (Q2 25 to $<50 \%$ ), moderate high (Q3 50 to $75 \%$ ), and high (Q4 $>75 \%)$.

For non-RRW, WTC exposure was grouped hierarchically into four levels based on the enrollee's eligibility category (local residents as the most exposed group, followed by area workers, passersby, and school staff/ students) [Brackbill et al., 2009], combined with qualitative exposure data (see Table SII). High exposure level applied to residents who did not evacuate or who returned home within the first 30 days after being evacuated on $9 / 11$, and who also reported exposure to the dust/debris cloud on 9/11. Low exposure (level 1) was assigned to persons who were not exposed to the dust/debris cloud, were not residents, and did not return to a lower Manhattan workplace if they were area workers or were not present at school on 9/11 if they were school staff/students.

Covariates included age, sex, race, and ethnicity, along with measures reported at enrollment: education; household income; smoking status; enrollment source; and history of asthma, cardiovascular disease, stroke, emphysema, or diabetes.

\section{Statistical Analyses}

RRW and non-RRW generally experienced different exposures and had very different demographic characteristics, and were therefore, analyzed separately in external and internal comparisons as explained below.

\section{External Comparisons}

To assess whether the number of cancer cases in the exposed population was greater or less than expected, we compared each group to the New York State (NYS) population using the standardized incidence ratio (SIR). The observed cancer cases were the sum of first primary incident cancers diagnosed during the period of 2007 through 2011. Expected cancer cases were determined from the NYS cancer rates and person-years of follow-up during 2007-2011, adjusted for age (5-year groups), race/ethnicity, sex, and calendar period (2007-2011). Race/ethnicity included non-Hispanic white, non-Hispanic black, Asian, Hispanic, and American Indian or Alaska Native. Rates from the NYS general population were used to calculate the number of cancer cases that would have been expected in the study population had they developed cancer at the NYS rate. The NYS cancer rates were chosen as the reference rate because NYS residents account for $86 \%$ of our study population. The NYS cancer rates used in these analyses were customized by NYS Department of Health to be comparable to our data based on stratification by age, sex, race/ ethnicity, and study period. Person-years of observation for each participant began on January 1,2007 and ended on the date of the first primary cancer diagnosis, death, withdrawal, or December 31, 2011, whichever came first. The number of expected cases was the sum of stratum-specific expected numbers of cancer cases that were products of stratum-specific rates and person-years. The 95\% confidence intervals (CI) for SIRs were calculated using Byar's approximation to the exact Poisson distribution [Breslow and Day, 1987]. All SIR computations were performed using MP-SIR sessions of SEER* ${ }^{*}$ STAT software (seer.cancer.gov/seerstat, version 7.0.5).

Finally, cancer rates based on the NYS population may not be generalizable to the other 10 states. To remove a possible source of under-representation we repeated the analyses, limiting the study population to enrollees residing continuously within NYS ( $\mathrm{n}=44,892 ; 18,171 \mathrm{RRW}$; and 26,721 non-RRW). A further sensitivity analysis was performed in enrollees residing in NYC compared to the NYC population.

\section{Internal Comparisons}

Comparisons among exposure groups were examined using Cox proportional hazards models separately for RRW and non-RRW participants. First, the cancers with significantly elevated SIRs in the study period were examined. Second, hematological cancers, which include myeloma, leukemia, and lymphoma, were examined as each has 
potentially shorter latency period compared to solid tumors, and thus, might be more likely to show an early increase. Third, lung cancer and bladder cancer were examined because carcinogens such as silica, asbestos, PAHs, and cadmium, detected in measurable quantities at the WTC site have been associated with both cancers.

Violation of the proportional hazards assumption was tested by including time dependent covariates in the Cox model and indicated by their significance. Hazard ratios (HR) and $95 \%$ confidence intervals were adjusted for age at enrollment, sex, race/ethnicity, smoking, education, income, and history of a serious non-malignant medical condition as described above. Enrollment source (self- vs. list-identified) was included in multivariate analyses to control for potential selection bias.

\section{Assessing Potential Medical Screening Bias}

Individual cancer screening practice may introduce bias in the number of observed cancer cases. Lacking data on medical screening of participants, we indirectly assessed potential screening bias by comparing the proportion of localized cancers in our sample to that in the NYS general population based on the same inclusion criteria (i.e., NYS residents, the same time period of 2007-2011, age $\geq 20$ years at diagnosis, and availability of race/ethnicity and stage), on the assumption that screening-detected cancers are more likely to be localized cancers. We focused on prostate, thyroid, and female breast cancers widely thought to be over-diagnosed through screening [Welch and Black, 2010]. Proportions were compared using Pearson's chi-squared test.

All descriptive and multivariate analyses were performed using SAS software (SAS Institute, Cary, NC, version 9.2). Significance was set at $P<0.05$ (two-sided).

\section{RESULTS}

\section{Baseline Characteristics}

The 60,339 enrollees (24,863 RRWs and 35,476 nonRRWs) meeting inclusion criteria for external comparison accounted for $84.5 \%$ of the cohort. After excluding 981 enrollees with unknown WTC exposure data, 59,358 enrollees remained for internal comparison (24,170 RRWs and 35,188 non-RRWs) (Fig. 1).

Table I shows characteristics of the study population by RRW status. Median age at enrollment was 42.0 years for RRW and non-RRW. RRWs were predominantly male (79.8\%), non-Hispanic white $(71.2 \%)$, and in households with income of $\$ 50,000$ to $<\$ 150,000(61.9 \%)$ at enrollment. Among non-RRWs, $46.9 \%$ were males, $57.9 \%$ non-Hispanic

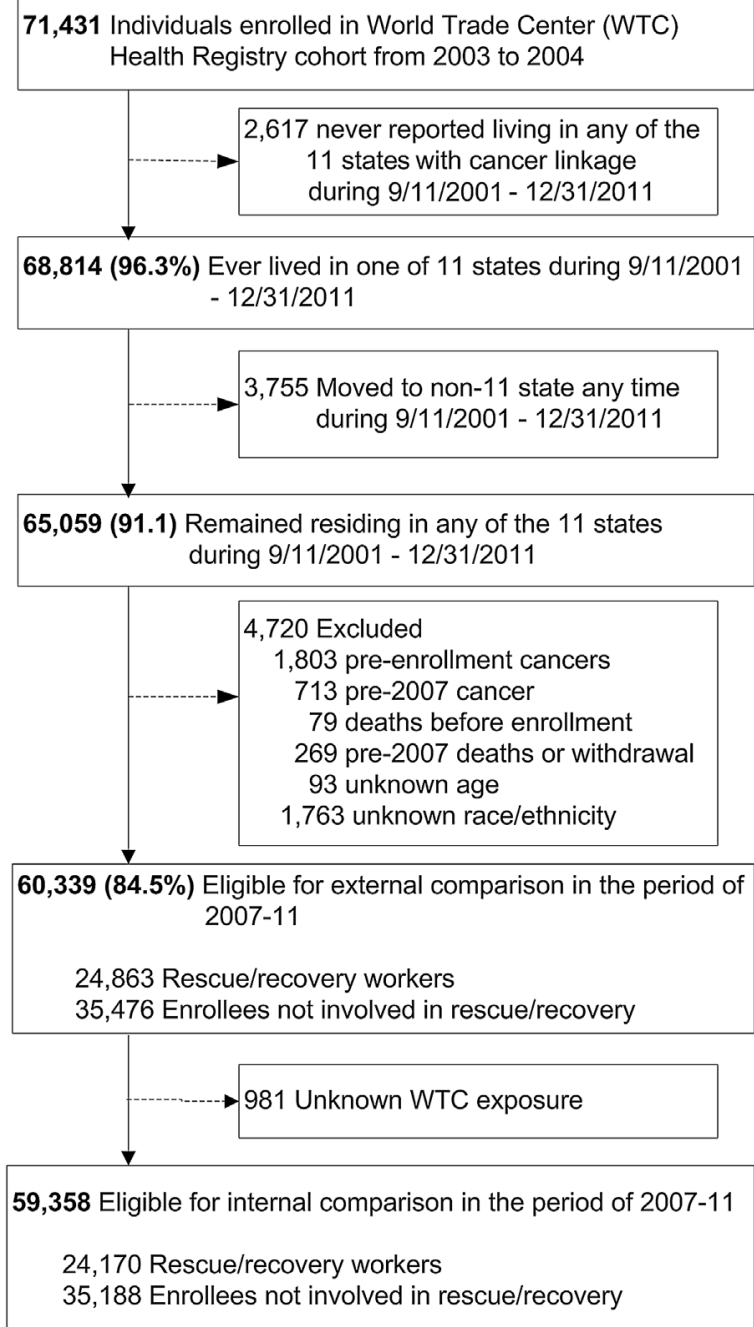

FIGURE 1. Selection of study population.

white, and $40.2 \%$ had annual household income of $\$ 50,000$ to $<\$ 150,000$ at enrollment.

\section{Cancer Incidence Among RRWs}

Of 24,863 eligible RRWs for external comparison using SIR, 685 first primary incident cancer cases were identified during 2007-2011, with an accumulated 120,876 personyears of follow-up. Median age at diagnosis was 57 years (range: $28-82$ years).

RRW had increased cancer incidence of all cancer sites combined that was significantly different from that in the reference population (SIR 1.11, 95\% CI $=1.03-1.20$ ) (Table II). Site-specific analyses showed 3 of the 24 cancer sites with significantly elevated SIR: prostate (\#observed 223, SIR 1.43, 95\% CI 1.25-1.63), thyroid (\#observed 37, SIR 1.79, 95\% CI 1.26-2.47), and skin melanoma (\#observed 37, SIR 1.49, 95\% CI 1.05-2.06). 
TABLE I. Characteristics of Study Enrollees by Rescue/Recovery Worker Status, WTCHR ( $N=60,339)$

\begin{tabular}{|c|c|c|c|c|}
\hline & \multicolumn{2}{|c|}{$\begin{array}{c}\text { Rescue/recovery workers } \\
\text { (RRW) }(\mathrm{n}=\mathbf{2 4 , 8 6 3 )}\end{array}$} & \multicolumn{2}{|c|}{$\begin{array}{l}\text { Enrollees not involved in rescue/recovery } \\
\qquad \text { (non-RRW) }(\mathrm{n}=\mathbf{3 5 , 4 7 6 )}\end{array}$} \\
\hline & $\#$ & $\%$ & \# & $\%$ \\
\hline \multicolumn{5}{|l|}{ Source of enrollment } \\
\hline List-identified & 8,418 & 33.9 & 8,523 & 24.0 \\
\hline Self-identified & 16,445 & 66.1 & 26,953 & 76.0 \\
\hline \multicolumn{5}{|l|}{ Age at enrollment, years } \\
\hline$<20$ & 71 & 0.3 & 2,797 & 7.9 \\
\hline $20-34$ & 5,204 & 20.9 & 7,883 & 22.2 \\
\hline $35-44$ & 9,412 & 37.9 & 8,973 & 25.3 \\
\hline $45-64$ & 9,684 & 38.9 & 13,963 & 39.4 \\
\hline$\geq 65$ & 492 & 2.0 & 1,860 & 5.2 \\
\hline Median & 42.0 & & 42.0 & \\
\hline \multicolumn{5}{|l|}{ Sex } \\
\hline Male & 19,848 & 79.8 & 16,631 & 46.9 \\
\hline Female & 5,015 & 20.2 & 18,845 & 53.1 \\
\hline \multicolumn{5}{|l|}{ Race/ethnicity } \\
\hline Non-Hispanic white & 17,704 & 71.2 & 20,553 & 57.9 \\
\hline Non-Hispanic black & 2,420 & 9.7 & 5,305 & 15.0 \\
\hline Hispanic & 3,823 & 15.4 & 5,028 & 14.2 \\
\hline Asian & 730 & 2.9 & 4,447 & 12.5 \\
\hline American Indian or Alaska Native & 186 & 0.7 & 143 & 0.4 \\
\hline \multicolumn{5}{|l|}{ Education completed at enrollment } \\
\hline Below high school & 1,375 & 5.5 & 4,165 & 11.7 \\
\hline High school & 6,337 & 25.5 & 5,075 & 14.3 \\
\hline Some college & 7,746 & 31.2 & 6,549 & 18.5 \\
\hline College or graduate & 9,318 & 37.5 & 19,520 & 55.0 \\
\hline Unknown & 87 & 0.3 & 167 & 0.5 \\
\hline \multicolumn{5}{|l|}{ Household income in 2002, \$ } \\
\hline$<50,000$ & 5,939 & 23.9 & 11,847 & 33.4 \\
\hline 50,000 to $<150,000$ & 15,399 & 61.9 & 14,249 & 40.2 \\
\hline$\geq 150,000$ & 1,594 & 6.4 & 5,113 & 14.4 \\
\hline Unknown & 1,931 & 7.8 & 4,267 & 12.0 \\
\hline \multicolumn{5}{|l|}{ Smoking status at enrollment } \\
\hline Current & 4,464 & 18.0 & 5,070 & 14.3 \\
\hline Former & 6,600 & 26.5 & 8,489 & 23.9 \\
\hline Never & 13,728 & 55.2 & 20,313 & 57.3 \\
\hline Unknown & 71 & 0.3 & 1,604 & 4.5 \\
\hline \multicolumn{5}{|l|}{ Any medical condition at enrollment ${ }^{a}$} \\
\hline Yes & 5,383 & 21.7 & 8,602 & 24.2 \\
\hline No/unknown & 19,480 & 78.3 & 26,874 & 75.8 \\
\hline \multicolumn{5}{|l|}{ Residence since September 11, 2001} \\
\hline New York state & 18,171 & 73.1 & 26,721 & 75.3 \\
\hline Any of the other 10 states & 4,446 & 17.9 & 4,361 & 12.3 \\
\hline Both & 2,246 & 9.0 & 4,394 & 12.4 \\
\hline \multicolumn{5}{|c|}{ Exposure level at WTC sites in rescue/recovery workers ${ }^{b}$} \\
\hline Low & 6,057 & 24.4 & NA & \\
\hline Intermediate & 6,208 & 25.0 & NA & \\
\hline Moderate high & 5,866 & 23.6 & NA & \\
\hline
\end{tabular}


TABLE I. (Continued)

\begin{tabular}{|c|c|c|c|c|}
\hline & \multicolumn{2}{|c|}{$\begin{array}{l}\text { Rescue/recovery workers } \\
\text { (RRW) }(\mathrm{n}=\mathbf{2 4 , 8 6 3 )}\end{array}$} & \multicolumn{2}{|c|}{$\begin{array}{l}\text { Enrollees not involved in rescue/recovery } \\
\qquad \text { (non-RRW) }(\mathrm{n}=\mathbf{3 5 , 4 7 6 )}\end{array}$} \\
\hline & \# & $\%$ & \# & $\%$ \\
\hline High & 6,039 & 24.3 & NA & \\
\hline No/unknown & 693 & 2.8 & NA & \\
\hline \multicolumn{5}{|c|}{ 9/11 exposure level in enrollees not involved in rescue/recovery ${ }^{b}$} \\
\hline Low & NA & & 2,537 & 7.2 \\
\hline Intermediate & NA & & 14,981 & 42.2 \\
\hline Moderate high & NA & & 13,444 & 37.9 \\
\hline High & NA & & 4,226 & 11.9 \\
\hline Unknown & NA & & 288 & 0.8 \\
\hline
\end{tabular}

WTCHR, World Trade Center Health Registry; NA, not available.

${ }^{a}$ Any history of heart disease, hypertension, angina, heart attack, stroke, diabetes, or emphysema reported at enrollment.

${ }^{b}$ See Supplemental Tables I and II for explanation.

A significantly reduced SIR was observed for lung cancer during the same follow-up period (\#observed 42, SIR 0.69, 95\% CI 0.50-0.93). One mesothelioma case was reported.

When the analyses were limited to the 18,171 NYS residents, SIRs remained significantly elevated for prostate (\#observed 165, SIR 1.48, 95\% CI 1.27-1.73), thyroid (\#observed 27, SIR 1.87, 95\% CI 1.24-2.73), and skin melanoma (\# observed 28, SIR 1.63, 95\% CI 1.08-2.35) and were depressed for lung cancer (\#observed 29, SIR 0.70, 95\% CI 0.47-1.00).

When the analyses were further limited to NYC residents, the SIR became significantly elevated for kidney cancer (\#observed 19, SIR 1.82, 95\% CI 1.08-2.88), while other SIRs remained the same.

\section{Cancer Incidence Among Non-RRWs}

A total of 992 first primary incident cancer cases were identified among 35,476 non-RRWs, with an accumulated 171,901 person-years of follow-up. Median age at diagnosis was 59 years (range: 7-99 years); two cancers were diagnosed in persons $<20$ years old.

Cancer incidence for all sites combined (Table II) among non-RRW was also significantly greater than the reference population during the follow-up period from 2007 through 2011 (SIR $=1.08,95 \%$ CI 1.02-1.15). Four of the 24 cancer sites were significantly elevated: prostate (\#observed 196, SIR $1.27,95 \%$ CI 1.10-1.46), skin melanoma (\#observed 44, SIR 1.54, 95\% CI 1.12-2.07), female breast (\#observed 187, SIR 1.34, 95\% CI 1.15-1.55), and non-Hodgkin's lymphoma (\#)bserved $=56, \mathrm{SIR}=1.49,95 \%$ CI 1.13-1.93).

A significantly reduced SIR was also observed for lung cancer during the same follow-up period (\#observed 67, SIR $0.69,95 \%$ CI $0.54-0.88$ ). One mesothelioma case was reported among non-RRW.
In the subgroup of 26,721 non-RRW who had been NYS residents since 9/11, SIRs remained significant for female breast (\#observed 150, SIR 1.32, 95\% CI 1.12-1.55) and non-Hodgkin's lymphoma (\#observed 44, SIR 1.52, 95\% CI 1.11-2.04). SIRs for prostate cancer and skin melanoma became non-significant. When further limited to NYC residents compared to the NYC population, SIRs for the four cancer sites remained significantly elevated.

\section{Internal Comparisons by Level of Exposure Among RRWs}

Table III shows adjusted hazard ratios (aHR) for selected cancers among RRW, adjusting for baseline characteristics and enrollment source. Compared with the lowest WTC exposure level, hazard ratios at each exposure quartile were not significantly elevated for all sites-combined or any site-specific cancer. However, urinary bladder cancer was significantly associated with increased WTC exposure in the $\log$ transformation (aHR 2.18, 95\% CI 1.10-4.34, $P=0.0259$ ), while the dose-response trend for urinary bladder cancer using the original WTC exposure score was not (aHR 1.03, 95\% CI 0.99-1.05, $P=0.1098$ ). No significant dose-response trend was observed for hematologic cancers among RRWs.

In a sub-analysis of kidney cancer among RRWs who were NYC residents, there was no significant dose-response trend associated with kidney cancer and WTC exposure levels.

\section{Internal Comparisons by Level of Exposure Among Non-RRWs}

Table IV shows aHR for selected cancers among nonRRWs after adjusting for baseline characteristics and source 
TABLE II. Standardized Incidence Ratio (SIR) of First Primary Cancer Site Among Eligible Enrollees Using the New York State Population Rate as Reference, 2007-2011 ( $=60,339)$

\begin{tabular}{|c|c|c|c|c|c|c|c|c|}
\hline \multirow[b]{2}{*}{ Site (SEER recode) ${ }^{b}$} & \multicolumn{4}{|c|}{ Rescue/recovery workers (RRW) $(\mathrm{N}=\mathbf{2 4 , 8 6 3 )}$} & \multicolumn{4}{|c|}{$\begin{array}{l}\text { Enrollees not involved in rescue and recovery } \\
\qquad \text { (non-RRW) }(\mathrm{N}=\mathbf{3 5 , 4 7 6 )}\end{array}$} \\
\hline & No. observed & No. expected & $\operatorname{SIR}^{\mathrm{a}}$ & (95\% CI) & No. observed & No. expected & $\operatorname{SIR}^{\mathrm{a}}$ & (95\% Cl) \\
\hline All sites combined & 685 & 617 & 1.11 & $(1.03-1.20)^{*}$ & 992 & 916 & 1.08 & $(1.02-1.15)^{*}$ \\
\hline Oral cavity and pharynx (20010-20100) & 16 & 20 & 0.80 & $(0.46-1.30)$ & 16 & 22 & 0.73 & $(0.42-1.19)$ \\
\hline Esophagus (21010) & 10 & 8 & 1.29 & $(0.62-2.38)$ & 7 & 9 & 0.80 & $(0.32-1.65)$ \\
\hline Stomach (21020) & 9 & 10 & 0.89 & $(0.41-1.68$ & 10 & 16 & 0.61 & $(0.29-1.12)$ \\
\hline Colorectal (21041-21052) & 47 & 51 & 0.93 & $(0.68-1.24)$ & 74 & 75 & 0.98 & $(0.77-1.23)$ \\
\hline Liver and intrahepatic bile duct (21071-21072) & 13 & 15 & 0.86 & $(0.46-1.46)$ & 27 & 19 & 1.41 & $(0.93-2.05)$ \\
\hline Pancreas (21100) & 10 & 14 & 0.70 & $(0.34-1.29)$ & 22 & 22 & 1.02 & $(0.64-1.54)$ \\
\hline Larynx (22020) & $\leq 5$ & 7 & 0.55 & $(0.15-1.42)$ & 9 & 8 & 1.18 & $(0.54-2.25)$ \\
\hline Lung and bronchus (22030) & 42 & 61 & 0.69 & $(0.50-0.93)^{*}$ & 67 & 97 & 0.69 & $(0.54-0.88)^{*}$ \\
\hline Melanoma of the skin (25010) & 37 & 25 & 1.49 & $(1.05-2.06)^{*}$ & 44 & 29 & 1.54 & $(1.12-2.07)^{*}$ \\
\hline Female breast (26000) & 48 & 39 & 1.22 & $(0.90-1.61)$ & 187 & 140 & 1.34 & $(1.15-1.55)^{*}$ \\
\hline Corpus uterus and NOS $(27020,27030)$ & 8 & 10 & 0.82 & $(0.35-1.62)$ & 37 & 36 & 1.03 & $(0.72-1.41)$ \\
\hline Ovary (27040) & $\leq 5$ & $\leq 5$ & 0.80 & $(0.16-2.33)$ & 13 & 14 & 0.95 & $(0.51-1.63)$ \\
\hline Prostate (28010) & 223 & 156 & 1.43 & $(1.25-1.63)^{*}$ & 196 & 154 & 1.27 & $(1.10-1.46)^{*}$ \\
\hline Testis (28020) & 7 & 8 & 0.92 & $(0.37-1.91)$ & 6 & $\leq 5$ & 1.12 & $(0.41-2.43)$ \\
\hline Urinary bladder (29010) & 30 & 27 & 1.10 & $(0.74-1.57)$ & 30 & 34 & 0.89 & $(0.60-1.27)$ \\
\hline Kidney and renal pelvis (29020) & 35 & 26 & 1.35 & $(0.94-1.88)$ & 36 & 30 & 1.20 & $(0.84-1.66)$ \\
\hline Brain and other nervous system $(31010,31040)$ & 7 & 9 & 0.77 & $(0.31-1.59)$ & 15 & 11 & 1.32 & $(0.74-2.18)$ \\
\hline Thyroid (32010) & 37 & 21 & 1.79 & $(1.26-2.47)^{*}$ & 48 & 39 & 1.23 & $(0.91-1.64)$ \\
\hline Hodgkin’s lymphoma (33011-33012) & 8 & $\leq 5$ & 1.79 & $(0.77-3.53)$ & $\leq 5$ & $\leq 5$ & 0.92 & $(0.30-2.14)$ \\
\hline Non-Hodgkin’s lymphoma (33041-33042) & 27 & 29 & 0.94 & $(0.62-1.37)$ & 56 & 38 & 1.49 & $(1.13-1.93)^{*}$ \\
\hline Multiple myeloma (34000) & 12 & 9 & 1.35 & $(0.70-2.36)$ & 9 & 13 & 0.67 & $(0.31-1.28)$ \\
\hline Leukemia (35011-35043) & 16 & 17 & 0.95 & $(0.54-1.54)$ & 18 & 22 & 0.81 & $(0.48-1.29)$ \\
\hline Soft tissue including heart (24000) & $\leq 5$ & $\leq 5$ & 0.67 & $(0.14-1.95)$ & $\leq 5$ & 6 & 0.33 & $(0.04-1.20)$ \\
\hline Mesothelioma (36010) & $\leq 5$ & $\leq 5$ & 1.02 & $(0.03-5.68)$ & $\leq 5$ & $\leq 5$ & 0.77 & $(0.02-4.28)$ \\
\hline
\end{tabular}

WTCHR, World Trade Center Health Registry; Cl, confidence interval.

${ }^{*} P$-value $<0.05$.

${ }^{a}$ Adjusted for age, gender, race/ethnicity, and study years.

${ }^{\mathrm{b}}$ Not all of the specific cancer sites were presented. Therefore, the number of all cancer sites combined is greater than the sum of specified cancer sites in the table.

of enrollment. There was a significant dose-response trend for melanoma in relation to exposure (aHR $1.53,95 \% \mathrm{CI}$ 1.04-2.23, $P=0.0300$ ), although the risks at the individual exposure levels were not statistically significant. No significant dose-response trend was observed for other sites.

\section{Localized Cancer}

Proportions of localized cancer in the NYS general adult population were $84.7 \%(55,097 / 65,026)$ for prostate, $68.0 \%$ $(9,871 / 14,508)$ for thyroid, and $63.0 \%$ for female breast cancer. We observed similar proportions of localized cancer for prostate cancer $(89.0 \%, 138 / 155)$, thyroid cancer $(52.0 \%$, $14 / 27)$, and female breast cancer $(65.6 \%, 21 / 32)$ among RRWs who were NYS residents since 9/11 $(P>0.05$, respectively). We further observed similar proportions of localized cancers among non-RRWs who were NYS residents since 9/11: $81.4 \%$ (96/118) for prostate, $79.4 \%$ (27/34) for thyroid, and $64.8 \%$ (94/145) for female breast cancer. None of these proportions was significantly different from those of the NYS general population $(P>0.05)$.

\section{DISCUSSION}

We observed a statistically significant excess incidence of total cancers among workers engaged in rescue/recovery operations between 9/11/2001 and 7/31/2002, and non-RRW survivors. Prostate cancer and malignant melanoma of the skin were significantly elevated in both populations, with prostate cancers contributing substantially to the total excess 
TABLE III. Risk of First Primary Cancer as a Function of WTC Exposure Among Rescue/Recovery Workers (RRW) With Known WTC Exposure Data, 2007-2011 ( $\mathrm{N}=24,170)$

\begin{tabular}{|c|c|c|c|c|c|}
\hline Site (SEER recode) & $\begin{array}{c}\text { WTC } \\
\text { exposure } \\
\text { score }\end{array}$ & $\begin{array}{l}\text { No. with first } \\
\text { incident cancer }\end{array}$ & $\begin{array}{l}\text { Person-years of } \\
\text { follow-up }\end{array}$ & $\begin{array}{l}\text { Adjusted hazard } \\
\text { ratio (aHR) }\end{array}$ & $\begin{array}{l}95 \% \text { confidence } \\
\text { interval (CI) }\end{array}$ \\
\hline \multirow{6}{*}{ All sites combined } & Low (Q1) & 177 & 29,304 & Ref & \\
\hline & $\begin{array}{l}\text { Intermediate } \\
\text { (Q2) }\end{array}$ & 171 & 30,212 & 1.03 & $0.83-1.27$ \\
\hline & $\begin{array}{l}\text { Moderate high } \\
\text { (Q3) }\end{array}$ & 162 & 28,538 & 1.08 & $0.87-1.35$ \\
\hline & High (Q4) & 147 & 29,487 & 1.13 & $0.89-1.44$ \\
\hline & Continuous & & & 1.00 & $0.997-1.01$ \\
\hline & $\log ^{\mathrm{C}}$ & & & 1.12 & $0.97-1.28$ \\
\hline \multirow[t]{6}{*}{ Lung and bronchus (22030) } & 01 & 12 & 29,304 & Ref & \\
\hline & Q2 & 8 & 30,212 & 0.71 & $0.29-1.77$ \\
\hline & Q3 & 15 & 28,538 & 1.53 & $0.69-3.43$ \\
\hline & Q4 & 7 & 29,487 & 0.78 & $0.28-2.16$ \\
\hline & Continuous & & & 1.00 & $0.97-1.03$ \\
\hline & $\log ^{c}$ & & & 1.13 & $0.67-1.94$ \\
\hline \multirow[t]{6}{*}{ Melanoma of the skin (25010) } & 01 & 10 & 29,304 & Ref & \\
\hline & Q2 & 6 & 30,212 & 0.73 & $0.26-2.07$ \\
\hline & Q3 & 9 & 28,538 & 1.16 & $0.43-3.09$ \\
\hline & Q4 & 10 & 29,487 & 1.18 & $0.42-3.34$ \\
\hline & Continuous & & & 1.01 & $0.98-1.03$ \\
\hline & $\log ^{c}$ & & & 1.05 & $0.59-1.89$ \\
\hline \multirow[t]{6}{*}{ Female breast (26000) } & 01 & 21 & 10,383 & Ref & \\
\hline & Q2 & 13 & 6,870 & 0.96 & $0.46-1.97$ \\
\hline & Q3 & 10 & 4,953 & 1.16 & $0.52-2.62$ \\
\hline & Q4 & 3 & 1,454 & 1.70 & $0.49-5.98$ \\
\hline & Continuous & & & 1.02 & $0.98-1.05$ \\
\hline & $\log ^{\mathrm{C}}$ & & & 1.16 & $0.65-2.05$ \\
\hline \multirow[t]{6}{*}{ Prostate (28010) } & Q1 & 50 & 18,921 & Ref & \\
\hline & Q2 & 61 & 23,342 & 1.17 & $0.80-1.71$ \\
\hline & Q3 & 49 & 23,585 & 0.99 & $0.66-1.49$ \\
\hline & Q4 & 54 & 28,034 & 1.25 & $0.82-1.89$ \\
\hline & Continuous & & & 1.01 & $0.995-1.02$ \\
\hline & $\log ^{\mathrm{C}}$ & & & 1.17 & $0.92-1.49$ \\
\hline \multirow[t]{6}{*}{ Urinary bladder (29010) } & Q1 & 0 & 29,304 & NA & \\
\hline & Q2 & 10 & 30,212 & Ref & \\
\hline & Q3 & 10 & 28,538 & 1.21 & $0.50-2.94$ \\
\hline & Q4 & 7 & 29,487 & 0.87 & $0.31-2.44$ \\
\hline & Continuous & & & 1.03 & $0.995-1.05$ \\
\hline & $\log ^{\mathrm{C}}$ & & & $2.18^{*}$ & $1.10-4.34$ \\
\hline \multirow[t]{6}{*}{ Thyroid (32010) } & Q1 & 9 & 29,304 & Ref & \\
\hline & Q2 & 9 & 30,212 & 0.98 & $0.38-2.52$ \\
\hline & Q3 & 8 & 28,538 & 0.93 & $0.34-2.52$ \\
\hline & Q4 & 11 & 29,487 & 1.35 & $0.50-3.64$ \\
\hline & Continuous & & & 1.00 & $0.97-1.02$ \\
\hline & $\log ^{c}$ & & & 1.08 & $0.61-1.92$ \\
\hline Hematological cancer ${ }^{\mathrm{b}}(35011-35043,33041$, & Q1 & 16 & 29,304 & Ref & \\
\hline $33042,33011-33012,34000)$ & Q2 & 14 & 30,212 & 0.85 & $0.41-1.77$ \\
\hline
\end{tabular}


TABLE III. (Continued)

\begin{tabular}{|c|c|c|c|c|c|}
\hline Site (SEER recode) & $\begin{array}{c}\text { WTC } \\
\text { exposure } \\
\text { score }\end{array}$ & $\begin{array}{l}\text { No. with first } \\
\text { incident cancer }\end{array}$ & $\begin{array}{l}\text { Person-years of } \\
\text { follow-up }\end{array}$ & $\begin{array}{l}\text { Adjusted hazard } \\
\text { ratio (aHR) }\end{array}$ & $\begin{array}{c}\text { 95\% confidence } \\
\text { interval (CI) }\end{array}$ \\
\hline & Q3 & 12 & 28,538 & 0.77 & $0.36-1.68$ \\
\hline & Q4 & 17 & 29,487 & 1.11 & $0.52-2.37$ \\
\hline & Continuous & & & 1.00 & $0.99-1.03$ \\
\hline & $\log ^{C}$ & & & 1.04 & $0.66-1.64$ \\
\hline \multirow[t]{6}{*}{ Non-Hodgkin's lymphoma $(33041,33042)$} & Q1 & 9 & 29,304 & Ref & \\
\hline & Q2 & 4 & 30,212 & 0.42 & $0.13-1.38$ \\
\hline & Q3 & 5 & 28,538 & 0.57 & $0.18-1.78$ \\
\hline & Q4 & 7 & 29,487 & 0.89 & $0.29-2.73$ \\
\hline & Continuous & & & 1.00 & $0.97-1.03$ \\
\hline & $\log ^{\mathrm{C}}$ & & & 0.91 & $0.45-1.83$ \\
\hline
\end{tabular}

NA, not available; Ref, referent.

${ }^{*} P$-value $=0.0259$.

${ }^{a}$ Cox proportional hazards modeling, adjusted for age at the beginning of the follow-up period, race/ethnicity, sex, household income in 2002, education, source of enrollment, smoking, and other medical conditions at enrollment.

Included leukemia, Hodgkin's and non-Hodgkin's lymphomas, and multiple myeloma.

${ }^{c} \log$ (natural) transformation of the actual WTC exposure scores.

of cancer across all sites. Thyroid cancer was significantly elevated only in RRW, while breast cancer and nonHodgkin's lymphoma were significantly elevated only in non-RRW. Lung cancer in both populations was significantly below expectation, by about $30 \%$.

These findings utilize cancer cases diagnosed from 2007 through 2011, and thus, extend our earlier work [Li et al., 2012] by 3 years, allowing the possibility of observation of cancers with longer latency periods within a population whose background cancer rates in general are rising due to aging. Excess prostate and thyroid cancers in this cohort were also observed during 2007-2008 [Li et al., 2012] among RRW but not among non-RRW. A significant excess of multiple myeloma among RRW in 2007-2008 was not sustained in 2007-2011. Excess breast cancer and non-Hodgkin's lymphoma are new findings among non-RRW not seen in the period of 2007-2008.

Although no comprehensive exposure measurements are available for this cohort, numerous known and possible carcinogens were identified in air and dust at the WTC site and throughout lower Manhattan [NIOSH, 2011, 2012]. These included asbestos and silica, metals such as cadmium and lead, PAH, dioxins, PCBs, and benzene [Wallingford and Snyder 2001; Lioy et al., 2002; McKinney et al., 2002; Edelman et al., 2003]. Intense and prolonged respiratory exposures to these dusts among RRW were likely, and numerous epidemiological studies have consistently found strong associations with early development of non-malignant respiratory diseases including asthma [Brackbill et al., 2009; Perlman et al., 2011]. Furthermore, potential exposures for rescue/recovery workers may have been exacerbated by fires that burned at the WTC site for months [Lioy et al., 2002], as well as by carcinogenic products of combustion, and pyrolysis products from burning building materials and furnishings. Six weeks after the attacks, exposure to polychlorinated dibenzo- $p$-dioxins and dibenzofurans (PCDD/Fs) through soil, and inhalation was up to 2.6 orders of magnitude higher in lower Manhattan than typical background levels measured in mid-Manhattan and Brooklyn [Rayne, 2005]. Cadmium in particular, an established carcinogen, has been associated with bladder cancer [FekiTounsi and Hamza-Chaffai, 2014], and melanoma [Venza et al., 2014], but inconsistently with prostate cancer [Waalkes and Rehm, 1994; Sahmoun et al., 2005]. In the present study, we observed a significant dose-response trend for WTC exposure level and urinary bladder cancer among RRW, and for skin melanoma among non-RRW, but not for other cancers with a statistically significant excess. Thus, the strength of the evidence for the elevated cancers being causally related to WTC exposure is limited.

The new findings among non-RRW are interesting, particularly the significant excess incidence of non-Hodgkin's lymphoma (SIR 1.49, 95\% CI 1.13-1.93). In addition, hazard ratios were greater in each of the three higher exposure groups in comparison with the lowest (reference) exposure, ranging from 1.37 to 1.84 , although none were statistically significant, possibly due to the small number of total cases (54) distributed among four exposure levels. A number of occupational studies reported a significant elevation of non-Hodgkin's lymphoma risk in persons with probable occupational exposure to benzene [Smith et al., 2007]. In view, however, of the relatively short follow-up period, and lack of data on other risk 
TABLE IV. Risk of First Primary Cancer as a Function of WTC Exposure Among Those Not Involved in Rescue/Recovery (Non-RRW) With Known WTC Exposure Data, 2007-2011 ( $\mathrm{N}=35,188)$

\begin{tabular}{|c|c|c|c|c|c|}
\hline Site (SEER recode) & $\begin{array}{c}\text { WTC } \\
\text { exposure } \\
\text { level }\end{array}$ & $\begin{array}{c}\text { No. with first } \\
\text { incident cancer }\end{array}$ & $\begin{array}{l}\text { Person-years of } \\
\text { follow-up }\end{array}$ & $\begin{array}{l}\text { Adjusted hazard } \\
\text { ratio (aHR) }^{\mathrm{a}}\end{array}$ & $\begin{array}{c}\text { 95\% confidence } \\
\text { interval (CI) }\end{array}$ \\
\hline \multirow[t]{5}{*}{ All sites combined } & $\operatorname{Low}(1)$ & 65 & 12,341 & Ref & \\
\hline & Intermediate (2) & 399 & 72,495 & 0.95 & $0.72-1.23$ \\
\hline & Moderate high (3) & 403 & 65,294 & 1.03 & $0.79-1.35$ \\
\hline & High (4) & 115 & 20,385 & 0.86 & $0.63-1.17$ \\
\hline & Trend (1-4) & & & 0.99 & $0.91-1.07$ \\
\hline \multirow[t]{5}{*}{ Female breast (26000) } & 1 & 14 & 5,373 & Ref & \\
\hline & 2 & 85 & 38,739 & 0.83 & $0.47-1.47$ \\
\hline & 3 & 68 & 35,118 & 0.68 & $0.38-1.21$ \\
\hline & 4 & 19 & 11,456 & 0.60 & $0.30-1.21$ \\
\hline & Trend (1-4) & & & 0.84 & $0.69-1.01$ \\
\hline Hematological cancer $^{\mathrm{b}}(35011-35043,33041$, & 1 & 6 & 12,341 & Ref & \\
\hline \multirow[t]{4}{*}{$33042,33011-33012,34000)$} & 2 & 33 & 72,495 & 0.83 & $0.34-2.01$ \\
\hline & 3 & 35 & 65,294 & 0.93 & $0.38-2.26$ \\
\hline & 4 & 12 & 20,385 & 0.89 & $0.32-2.45$ \\
\hline & Trend (1-4) & & & 1.02 & $0.78-1.33$ \\
\hline \multirow[t]{5}{*}{ Lung and bronchus (22030) } & 1 & 2 & 12,341 & Ref & \\
\hline & 2 & 30 & 72,495 & 1.64 & $0.39-7.03$ \\
\hline & 3 & 26 & 65,294 & 1.78 & $0.42-7.60$ \\
\hline & 4 & 8 & 20,385 & 1.15 & $0.24-5.58$ \\
\hline & Trend (1-4) & & & 0.96 & $0.70-1.29$ \\
\hline \multirow[t]{5}{*}{ Melanoma of the skin (25010) } & 1 & 2 & 12,341 & Ref & \\
\hline & 2 & 13 & 72,495 & 1.51 & $0.34-6.77$ \\
\hline & 3 & 20 & 65,294 & 2.66 & $0.60-11.72$ \\
\hline & 4 & 7 & 20,385 & 3.28 & $0.66-16.28$ \\
\hline & Trend (1-4) & & & $1.53^{*}$ & $1.04-2.23$ \\
\hline \multirow[t]{5}{*}{ Non-Hodgkin’s lymphoma $(33041,33042)$} & 1 & 2 & 12,341 & Ref & \\
\hline & 2 & 19 & 72,495 & 1.37 & $0.31-5.96$ \\
\hline & 3 & 25 & 65,294 & 1.84 & $0.43-7.94$ \\
\hline & 4 & 8 & 20,385 & 1.68 & $0.34-8.17$ \\
\hline & Trend (1-4) & & & 1.18 & $0.84-1.66$ \\
\hline \multirow[t]{5}{*}{ Prostate (28010) } & 1 & 16 & 6,967 & Ref & \\
\hline & 2 & 75 & 33,756 & 0.89 & $0.51-1.54$ \\
\hline & 3 & 87 & 30,176 & 1.02 & $0.58-1.77$ \\
\hline & 4 & 17 & 8,928 & 0.69 & $0.34-1.40$ \\
\hline & Trend (1-4) & & & 0.96 & $0.80-1.15$ \\
\hline \multirow[t]{5}{*}{ Thyroid (32010) } & 1 & 1 & 12,341 & Ref & \\
\hline & 2 & 20 & 72,495 & 3.49 & $0.47-26.22$ \\
\hline & 3 & 24 & 65,294 & 4.18 & $0.56-31.35$ \\
\hline & 4 & 3 & 20,385 & 1.97 & $0.20-19.21$ \\
\hline & Trend (1-4) & & & 1.08 & $0.74-1.56$ \\
\hline \multirow[t]{5}{*}{ Urinary bladder (29010) } & 1 & 2 & 12,341 & Ref & \\
\hline & 2 & 15 & 72,495 & 1.05 & $0.23-4.77$ \\
\hline & 3 & 10 & 65,294 & 0.79 & $0.17-3.70$ \\
\hline & 4 & 2 & 20,385 & 0.41 & $0.06-3.10$ \\
\hline & Trend (1-4) & & & 0.74 & $0.46-1.19$ \\
\hline
\end{tabular}

NA, not available; Ref, referent.

${ }^{*} P$-value $=0.0300$.

${ }^{a}$ Cox proportional hazards modeling, adjusted for age at the beginning of the follow-up period, race/ethnicity, sex, household income in 2002, education, source of enrollment, smoking, and other medical conditions at enrollment.

'Included leukemia, Hodgkin's and non-Hodgkin's lymphomas, and multiple myeloma. 
factors for NHL, including immunosuppression and infectious agents (e.g., Epstein-Barr virus) [Müller et al., 2005], the findings from this study need to be further studied with more cases in a longer follow-up period.

Strengths of this study include linkage with cancer registries in states that are estimated to include at least $91 \%$ of WTCHR enrollees, and the large sample size that facilitated internal comparisons to examine whether any of the cancers with higher than expected incidence were associated with higher levels of exposure. Inclusion of nonRRW provided an opportunity for assessment of risks in two distinct populations with different types of exposure opportunities. Furthermore, despite lack of objective exposure measurements, we were able to construct robust exposure categories using extensive exposure history data (intensity and duration) from the baseline survey.

An important study limitation is our inability to distinguish cancers detected because of increased monitoring and screening of this population. This especially affects the two most prominent site-specific findings, prostate, and thyroid cancers, for which excessive medical screening or early detection may have resulted in an increase in incidence [Welch and Black, 2010; Brito and Davies, 2014; Davies and Welch, 2014]. Although, no significant differences were found in the proportion of localized (early stage) cancers between our study sample and the NYS population, the contrast between the significantly elevated SIR of 1.34 for female breast cancer among non-RRW with the nonsignificant adjusted hazard ratios below 1.0 in higher versus lower exposed sub-groups may be due to our inability to control for early cancer detection activities in these data such as number of examinations, use of advanced diagnostic imaging, or medical examinations leading to unintended cancer detection.

There are other limitations as well. It is likely that 10 years of follow-up is an insufficient latency period for some cancers. The small number of cases for some cancers due to relatively short follow-up period may also affect the stability of SIRs. The significant SIR for kidney cancer, when the study population was restricted to NYC residents, might have resulted from the $46 \%$ reduction in the number of cases. Further work is required to determine the etiologic significance of this observation.

Also, we lacked occupational histories for enrollees who may have been exposed to hazardous materials prior to $9 / 11$. This is especially true for firefighters, because employment in firefighting-related occupations has repeatedly been suggested as a risk factor for malignant skin melanoma, prostate, bladder cancer, multiple myeloma, non-Hodgkin's lymphoma, and kidney cancer [LeMasters et al., 2006; Bates 2007; Daniels et al., 2014; Tsai et al., 2015]. The SIR results may be biased by residual confounding because there are usually many other differences between the exposed population and the general population besides age, sex, and race/ethnicity [Rothman and Greenland, 2008]. For example, smoking is a well-known risk factor for lung cancer, and smoking prevalence in 2003-2004 was about 18\% for RRW and $14 \%$ for non-RRW, lower than the $19.9-21.6 \%$ in NYS adult population in 2003-2004 [NYSDH, 2006]. Therefore, the significant deficit of lung cancer seen in both groups may be due in part to the lower smoking rate in our study population. In addition, we lack information on some wellknown risk factors for cancer such as ultraviolet (UV) light exposure [Armstrong and Kricker, 2001], reproductive patterns [Althuis et al., 2005], and family history of cancer. Lastly, self-reported exposures could not be verified and were subject to recall, and reporting biases. However, given that exposure data were collected before the outcome cancer was diagnosed, the influence of recall and reporting biases on cancer outcomes should be minimal.

\section{CONCLUSIONS}

In this extended follow-up study, excess incidence of prostate and thyroid cancers continued among RRW while small but statistically significantly higher rates than expected were found for skin melanoma in both populations, and female breast cancer and non-Hodgkin's lymphoma among non-RRW. Only for lung cancer was there a significantly lower rate. The increased incidence in most of these cancers lacked support from internal comparisons to examine whether there was an association with magnitude of exposure. Even though a significant dose-response trend associated with melanoma among non-RRW was observed, the absence of information on a major confounder, that is, UV light exposure before and after 9/11 makes meaningful interpretation of causality between WTC exposure and the cancer difficult. Despite the limitations of the study, our findings show some evidence of increased risk of cancer among WTC exposed populations, yet they need to be substantiated by additional follow-up studies as the latency period from a potential WTC exposure increases. Longer term follow-up could be of direct value in describing the magnitude of any differences seen in comparison to the general population, and whether they might be related to WTC exposure during 9/11 and its aftermath.

\section{AUTHORS' CONTRIBUTIONS}

All authors made substantial contributions to the conception or design of the work; or the acquisition, analysis, or interpretation of data for the work; and drafted the work or revised it critically for important intellectual content; and provided final approval of the version to be published; and agreed to be accountable for all aspects of the work in ensuring that questions related to the accuracy or integrity of any part of the work are appropriately investigated and resolved. 


\section{ACKNOWLEDGMENTS}

We gratefully acknowledge the participation of Registry enrollees. We thank the 11 State Cancer Registries for carrying out record linkages: Bureau of Cancer Epidemiology, NYSDOH; California Cancer Registry, Department of Public Health (DPH); Connecticut Tumor Registry, DPH; Florida Cancer Registry, DOH; Massachusetts Cancer Registry, DPH; New Jersey State Cancer Registry, DOH; and Rutgers Cancer Institute of New Jersey; Ohio Cancer Incidence Surveillance System, DOH; Bureau of Health Statistics and Research, Pennsylvania DOH; Texas Cancer Registry, Texas Department of State Health Services, and Washington State Cancer Registry, DOH (See Supplemental acknowledgements). We also thank Maria J. Schymura of NYSDOH for her support; Steve Scoppa of Information Management Services, Inc., for his technical support of SEER* ${ }^{*}$ Stat software use; Rachel Zeig-Owens of the Montefiore Medical Center and Fire Department of the City of New York, Christopher D'Andrea of NYCDOHMH, and Vinicius Antao of Hospital for Special Surgery for their contributions to the WTC exposure matrix; Sharon Perlman, Charon Gwynn, Sukhminder Osahan, Rhoda Schlamm, and Margaret Millstone of NYCDOHMH for their review of the manuscript. None of the individuals received compensation for their contributions.

\section{FUNDING}

This publication was supported by Cooperative Agreement Number 5U50/OH009739 from the National Institute for Occupational Safety and Health (NIOSH) of the Centers for Disease Control and Prevention (CDC); U50/ ATU272750 from the Agency for Toxic Substances and Disease Registry (ATSDR), CDC, which included support from the National Center for Environmental Health, CDC; and by the New York City Department of Health and Mental Hygiene (NYC DOHMH). Its contents are solely the responsibility of the authors and do not necessarily represent the official views of NIOSH-CDC. Baozhen Qiao and Amy R. Kahn were supported by the New York State Department of Health (NYSDOH).

\section{DISCLOSURE (AUTHORS)}

The authors declare no conflicts of interest.

\section{DISCLOSURE BY AJIM EDITOR OF RECORD}

Steven Markowitz declares that he has no competing or conflicts of interest in the review and publication decision regarding this article.

\section{DISCLAIMER}

None.

\section{REFERENCES}

Althuis MD, Dozier JM, Anderson WF, Devesa SS, Brinton LA. 2005. Global trends in breast cancer incidence and mortality 1973-1997. Int J Epidemiol 34:405-412.

Antao VC, Pallos LL, Shim YK, Sapp JH 2nd, Brackbill RM, Cone JE, Stellman SD, Farfel MR. 2011. Respiratory protective equipment, mask use, and respiratory outcomes among World Trade Center rescue and recovery workers. Am J Ind Med 54(12):897-905.

Armstrong BK, Kricker A. 2001. The epidemiology of UV induced skin cancer. J Photochem Photobiol B 63:8-18.

Bates MN. 2007. Registry-based case-control study of cancer in California firefighters. Am J Ind Med 50:339-344.

Boffetta P, Zeig-Owens R, Wallenstein S, Li J, Brackbill R, Cone J, Farfel M, Holden W, Lucchini R, Webber MP, et al. 2016. Cancer in World Trade Center responders: Findings from multiple cohorts and options for future study. Am J Ind Med 59(2):96-105.

Brackbill RM, Hadler JL, DiGrande L, Ekenga CC, Farfel MR, Friedman S, Perlman SE, Stellman SD, Walker DJ, Wu D, et al. 2009. Asthma and posttraumatic stress symptoms 5 to 6 years following exposure to the World Trade Center terrorist attack. JAMA 302:502-516.

Breslow NE, Day NE. 1987. Statistical methods in cancer research. Vol. II, the design and analysis of cohort studies (IARC scientific publication No. 82). Lyon, France: International Agency for Research on Cancer. pp. 69-71.

Brito JP, Davies L. 2014. Is there really an increased incidence of thyroid cancer? Curr Opin Endocrinol Diabetes Obes 21:405-408.

Daniels RD, Kubale TL, Yiin JH, Dahm MM, Hales TR, Baris D, Zahm SH, Beaumont JJ, Waters KM, Pinkerton LE. 2014. Mortality and cancer incidence in a pooled cohort of US firefighters from San Francisco, Chicago, and Philadelphia (1950-2009). Occup Environ Med 71:388-397.

Davies L, Welch HG. 2014. Current thyroid cancer trends in the United States. JAMA Otolaryngol Head Neck Surg 140:317-322.

Edelman P, Osterloh J, Pirkle J, Caudill SP, Grainger J, Jones R, Blount B, Calafat A, Turner W, Feldman D, et al. 2003. Biomonitoring of chemical exposure among New York City firefighters responding to the World Trade Center fire and collapse. Environ Health Perspect 111:1906-1911.

Farfel M, DiGrande L, Brackbill R, Prann A, Cone J, Friedman S, Walker DJ, Pezeshki G, Thomas P, Galea S, et al. 2008. An overview of 9/11 experiences and respiratory and mental health conditions among World Trade Center Health Registry enrollees. J Urban Health 85:880-909.

Feki-Tounsi M, Hamza-Chaffai A. 2014. Cadmium as a possible cause of bladder cancer: A review of accumulated evidence. Environ Sci Pollut Res 21:10561-10573.

Hasson F, Keeney S, McKenna H. 2000. Research guidelines for the Delphi survey technique. J Adv Nurs 32:1008-1015.

Kleinman EJ, Christos PJ, Gerber LM, Reilly JP, Moran WF, Einstein AJ, Neugut AI. 2015. NYPD cancer incidence rates 1995-2014 encompassing the entire world trade center cohort. J Occup Environ Med 57:e101-e113.

Landrigan PJ, Lioy PJ, Thurston G, Berkowitz G, Chen LC, Chillrud SN, Gavett SH, Georgopoulos PG, Geyh AS, Levin S, et al. 2004. NIEHS World Trade Center Working Group. Health and environmental consequences of the World Trade Center disaster. Environ Health Perspect 112:731-739. 
Lauby-Secretan B, Loomis D, Grosse Y, El Ghissassi F, Bouvard V, Benbrahim-Tallaa L, Guha N, Baan R, Mattock H, Straif K, et al. 2013. WHO International Agency for Research on Cancer. Carcinogenicity of polychlorinated biphenyls and polybrominated biphenyls. Lancet Oncol 14(4):287-288.

LeMasters GK, Genaidy AM, Succop P, Deddens J, Sobeih T, BarrieraViruet H, Dunning K, Lockey J. 2006. Cancer risk among firefighters: A review and meta-analysis of 32 studies. J Occup Environ Med 48:1189-1202.

Li J, Cone JE, Kahn AR, Brackbill RM, Farfel MR, Greene CM, Hadler JL, Stayner LT, Stellman SD. 2012. Association between World Trade Center exposure and excess cancer risk. JAMA 308:2479-2488.

Lioy PJ, Weisel CP, Millette JR, Eisenreich S, Vallero D, Offenberg J, Buckley B, Turpin B, Zhong M, Cohen MD, et al. 2002. Characterization of the dust/smoke aerosol that settled east of the World Trade Center (WTC) in lower Manhattan after the collapse of the WTC 11 September 2001. Environ Health Perspect 110:703-714.

Lioy PJ, Pellizzari E, Prezant D. 2006. The World Trade Center aftermath and its effects on health: Understanding and learning through human-exposure science. Environ Sci Technol 40:6876-6885.

McKinney K, Benson S, Lempert A, Singal M, Wallingford K, Snyder E. 2002. Occupational exposures to air contaminants at the world trade center disaster Site-New York, September-October, 2001. Morb Mortal Wkly Rep 51:453-456.

Müller AM, Ihorst G, Mertelsmann R, Engelhardt M. 2005. Epidemiology of non-Hodgkin's lymphoma (NHL): trends, geographic distribution, and etiology. Ann Hematol 84(1):1-12.

Murphy J, Brackbill RM, Thalji L, Dolan M, Pulliam P, Walker DJ. 2007. Measuring and maximizing coverage in the World Trade Center Health Registry. Stat Med 26:1688-1701.

National Cancer Institute. SEER site recode ICD-O-3 (1/27/2003) definition. 2003. Available: http://seer.cancer.gov/siterecode/icdo3_ d01272003/ [accessed 22 March 2016].

National Institute for Occupational Safety and Health (NIOSH). Scientific and medical evidence related to cancer for the World Trade Center Health Program. 2011. Available: http://www.cdc.gov/niosh/ docs/2011-197/pdfs/2011-197.pdf [accessed 22 March 2016].

National Institute for Occupational Safety and Health (NIOSH). World Trade Center chemicals of potential concern and selected other chemical agents. 2012. Available: http://www.cdc.gov/niosh/docs/ 2012-115/pdfs/2012-115.pdf [accessed 22 March 2016].

New York State Department of Health. BRFSS Brief: cigarette smoking among New York State adults. 2006. Available: www.health.ny.gov/ statistics/brfss/reports/docs/brfssbrief_smoking_0707.pdf [accessed 22 March 2016].
Perlman SE, Friedman S, Galea S, Nair HP, Eros-Sarnyai M, Stellman SD, Hon J, Greene CM. 2011. Short-term and medium-term health effects of 9/11. Lancet 378:925-934.

Rayne S. 2005. Using exterior building surface films to assess human exposure and health risks from PCDD/Fs in New York City, USA, after the World Trade Center attacks. J Hazard Mater 127:33-39.

Rothman KJ, Greenland S, Lash TL. 2008. Modern Epidemiology, 3rd Edition. Philadelphia, PA: Lippincott, Williams \& Wilkins. pp. 67-69.

Sahmoun AE, Case LD, Jackson SA, Schwartz GG. 2005. Cadmium and prostate cancer: A critical epidemiologic analysis. Cancer Invest 23(3):256-263

Smith MT, Jones RM, Smith AH. 2007. Benzene exposure and risk of non-Hodgkin lymphoma. Cancer Epidemiol Biomarkers Prev 16(3): $385-391$.

Solan S, Wallenstein S, Shapiro M, Teitelbaum SL, Stevenson L, Kochman A, Kaplan J, Dellenbaugh C, Kahn A, Biro FN, et al. 2013. Cancer incidence in world trade center rescue and recovery workers, 2001-2008. Environ Health Perspect 121:699-704.

Tsai RJ, Luckhaupt SE, Schumacher P, Cress RD, Deapen DM, Calvert GM. 2015. Risk of cancer among firefighters in California, 1988-2007. Am J Ind Med 58(7):715-729.

Venza M, Visalli M, Biondo C, Oteri R, Agliano F, Morabito S, Caruso G, Caffo M, Teti D, Venza I. 2014. Epigenetic effects of cadmium in cancer: Focus on melanoma. Curr Genomics 15:420-435.

Waalkes MP, Rehm S. 1994. Cadmium and prostate cancer. J Toxicol Environ Health 43:251-269.

Wallingford KM, Snyder EM. 2001. Occupational exposures during the World Trade Center disaster response. Toxicol Ind Health 17:247-253.

Welch HG, Black WC. 2010. Overdiagnosis in cancer. J Natl Cancer Inst 102:605-613.

Zeig-Owens R, Webber MP, Hall CB, Schwartz T, Jaber N, Weakley J, Rohan TE, Cohen HW, Derman O, Aldrich TK, et al. 2011. Early assessment of cancer outcomes in New York City firefighters after the 9/11 attacks: An observational cohort study. Lancet 378: 898-905.

\section{SUPPORTING INFORMATION}

Additional supporting information may be found in the online version of this article at the publisher's web-site. 